\title{
Ejection Regimes in Picosecond Laser-Induced Forward Transfer of Metals
}

\author{
Ralph Pohl, ${ }^{1, *}$ Claas Willem Visser, ${ }^{2, \dagger}$ Gert-Willem Römer, ${ }^{1}$ Detlef Lohse, ${ }^{2}$ Chao Sun, ${ }^{2}$ and Bert Huis in 't Veld ${ }^{1}$ \\ ${ }^{1}$ Chair of Applied Laser Technology, Faculty of Engineering Technology, \\ University of Twente, Netherlands \\ ${ }^{2}$ Physics of Fluids Group, Faculty of Science and Technology, \\ J. M. Burgers Centre for Fluid Dynamics, University of Twente, Netherlands
}

(Received 18 July 2014; revised manuscript received 21 October 2014; published 3 February 2015)

\begin{abstract}
Laser-induced forward transfer (LIFT) is a 3D direct-write method suitable for precision printing of various materials, including pure metals. To understand the ejection mechanism and thereby improve deposition, here we present visualizations of ejection events at high-spatial (submicrometer) and hightemporal resolutions, for picosecond LIFT of copper and gold films with a thickness $50 \mathrm{~nm} \leq d \leq 400 \mathrm{~nm}$. For increasing fluences, these visualizations reveals the fluence threshold below which no ejection is observed, followed by the release of a metal cap (i.e., a hemisphere-shaped droplet), the formation of an elongated jet, and the release of a metal spray. For each ejection regime, the driving mechanisms are analyzed, aided by a two-temperature model. Cap ejection is driven by relaxation of thermal stresses induced by laser-induced heating, whereas jet and spray ejections are vapor driven (as the metal film is partly vaporized). We introduce energy balances that provide the ejection velocity in qualitative agreement with our velocity measurements. The threshold fluences separating the ejection regimes are determined. In addition, the fluence threshold below which no ejection is observed is quantitatively described using a balance between the surface energy and the inertia of the (locally melted) film. In conclusion, the ejection type can now be controlled, which allows for improved deposition of pure metal droplets and sprays.
\end{abstract}

DOI: 10.1103/PhysRevApplied.3.024001

\section{INTRODUCTION}

Laser-induced forward transfer (LIFT) is a highresolution 3D direct-write method that was first demonstrated in 1986 [1]. For the LIFT process, a transparent substrate (carrier) is coated with a thin film (donor) and is placed in close proximity to a second substrate (receiver); see Fig. 1. A pulsed laser beam is focused through the carrier onto the carrier-donor interface. The incident laser pulse is absorbed within a thin layer of the donor material. At sufficiently high laser fluences, the donor material is ejected and deposited onto a receiver substrate.

LIFT has a high potential for printing of various materials (including pure metals [2-7]) that cannot be deposited using conventional methods such as ink-jet printing, while retaining key advantages including high resolution (down to $300 \mathrm{~nm}$ [8]), and maskless, contact-free deposition at room conditions. In particular, the deposition of pure-metal droplets in the liquid phase allows for deposition of conductive patterns $[9,10]$, from which the semiconductor industry could benefit [11]. However, despite process improvements in various ways [12-16], the high potential of LIFT for liquid-metal deposition has not been met because the deposited features are poorly controlled. This lack of control can result in deposition of

\footnotetext{
*r.pohl@utwente.nl

†c.visser@utwente.nl
}

one main droplet surrounded by smaller satellite droplets, the deposition of many particles [8], or a significant uncertainty in the deposition location due to limited control of the ejection angle [17].

Improving LIFT is far from straightforward. The ejection process has hardly been visualized because of the extremely short time of the process and, consequently, the process is poorly understood. The ejection time scale is estimated to be only $\tau \sim V / L \sim 100 \mathrm{~ns}$, assuming a velocity $V \approx 100 \mathrm{~m} / \mathrm{s}$ and a length scale $L \approx 10 \mu \mathrm{m}$ [18], resulting in challenging visualization conditions. So far, time-resolved visualization has been achieved for relatively thick liquid-film [19-23] and solid-phase [24-26] or pastetransfer $[27,28]$ processes. Observations of LIFT processing of $\mathrm{Au}$ [29], $\mathrm{Ni}$ [30], $\mathrm{Al}$ [31], and $\mathrm{Cr}$ [32] do not provide sufficient spatial resolutions to track the process in detail.

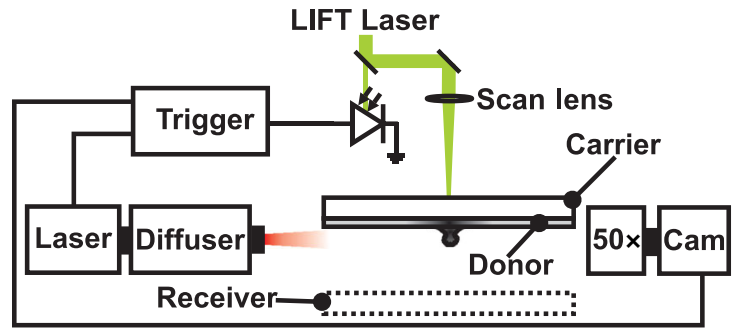

FIG. 1. Sketch of the experimental setup. The experiments were conducted without a receiving substrate. 
Therefore, theories describing the ejection mechanism have been proposed based on the craters left in the donor layer or deposited features on the receiver substrate [33-35]. In addition, numerical simulations have been performed [36-38]. Two driving mechanisms of the ejection process are commonly proposed (for these and more theories see Refs. [33,34,39]). First, relaxation of thermally induced stresses [40] could drive the ejection. Second, partial evaporation [39] of the donor layer, resulting in the formation of an expanding vapor bubble, may accelerate and eject the donor material. However, as yet, it is unknown under which conditions these ejection mechanisms occur.

Here we visualize and describe different types of ejections occurring in LIFT of copper and gold films. The determination of different ejection regimes allows optimization of the process parameters for specific applications, such as metal micromanufacturing. To this aim, high-speed, high-resolution visualization of ejection events in picosecond LIFT is pursued, revealing three ejection regimes and corresponding ejection velocities. We interpret the experimental results using a two-temperature model, providing key evidence for the underlying ejection mechanism. Based on this evidence, the energy balances required to model the ejection regime and ejection velocity are proposed. This approach provides the key parameter settings for each regime, and provides a simple estimate of the ejection-fluence threshold.

The experimental and numerical methods are discussed in Sec. II. High-resolution images of the ejection dynamics and two-temperature model calculations are presented in Sec. III, as well as the physical interpretation of these results. The implications and limitations of these results are discussed in Sec. IV, followed by the conclusions in Sec. V.

\section{METHODS}

\section{A. Experimental setup}

The experiments are performed using the setup schematically shown in Fig. 1. For LIFT, a Yb:YAG laser source was used with a fixed pulse duration of $6.7 \mathrm{ps}$, a wavelength of $515 \mathrm{~nm}$ second-harmonic generation (SHG), and a Gaussian beam profile with a beam quality factor of $M^{2} \leq 1.3$. The beam was focused onto the carrier-donor interface using a F-Theta-Ronar scan lens with a focal length of $100 \mathrm{~mm}$. The beam waist $\left(1 / e^{2}\right)$ was measured to be $8.3 \pm 0.6 \mu \mathrm{m}$. The fluence values in this paper represent peak fluences. The maximum error in the fluence is $20 \mathrm{~mJ} / \mathrm{cm}^{2}$, as determined using the $D^{2}$ method [41]. Copper and gold films with thicknesses of $50 \mathrm{~nm}<d<400 \mathrm{~nm}$ (magnetron sputtered at $23 \mathrm{~nm} / \mathrm{m}$ onto a 1-mm-thick glass carrier) are used as donor layer. To optimize the experimental imaging conditions, the receiving substrate was omitted.

Images were recorded using a dual-shot CCD camera (PCO Sensicam), mounted to a microscope with a $50 \times$ long-distance objective. Bright-field flash illumination was provided by a dual-cavity Nd:YAG laser with a pulse duration of $6 \mathrm{~ns}$. A high-efficiency diffuser was used to diffuse this laser pulse and thereby prevent fringes [42]. To determine the ejection velocity, the distance between the ejection crater in the donor layer and the tip of the ejection was measured and divided over the time between the ejection and the frame illumination. For triggering, a pulse-delay generator was used (Berkeley Nucleonics, BNC 575). The trigger sequence was started by the output of a photodiode exposed to the LIFT laser beam path. All components were selected to achieve high-temporal resolution, resulting in a temporal measurement error of $10 \mathrm{~ns}$. The spatial resolution of the imaging system is limited by the diffraction limit or motion blur (for ejection velocities exceeding $100 \mathrm{~m} / \mathrm{s}$ ).

\section{B. Two-temperature model}

As the laser pulse duration is comparable to the time scale of the electron-phonon relaxation [43], a two-temperature model (TTM) is used to describe the lattice temperature $T_{l}$ and the electron temperature $T_{e}$ of the donor layer. Hence, the temperature evolution of the electron and phonon subsystems is modeled by the following set of differential equations [44]:

$$
C_{e} \frac{\delta T_{e}}{\delta t}=\frac{\delta}{\delta z} K_{e} \frac{\delta T_{e}}{\delta z}-g\left(T_{e}-T_{l}\right)+S,
$$

and

$$
C_{l}\left(T_{l}\right) \frac{\delta T_{l}}{\delta t}=g\left(T_{e}-T_{l}\right),
$$

where $C_{e}, C_{l}\left(T_{l}\right)$, and $g$ represent the electron heat capacity, the phonon heat capacity, and the electron-phonon coupling factor, respectively (see the Supplemental Material [45]). The electron heat capacity is modeled as $C_{e}=A_{e} T_{e}$, where $A_{e}$ is the electron specific-heat constant, and the electron thermal conductivity as $K_{e}=K_{e 0} T_{e} / T_{l}$ with $K_{e 0}$ being the electron heat conductivity. The enthalpies of melting and vaporization are incorporated by adding a Gaussian function to the heat capacity, centered at the equilibrium phasechange temperatures, with a standard deviation of $20 \mathrm{~K}$. The integration of those functions yields the phase-change enthalpies. The source term $S$ describes the electron heating by Lambert-Beer absorption for thin films,

$$
S=\alpha(1-R) I_{0}(t) \frac{\exp (-\alpha z)}{1-\exp (-\alpha d)}
$$

where $R$ denotes the reflection coefficient and $\alpha$ the linear absorption coefficient. Transmitted light is excluded by the denominator in Eq. (3) $[1-\exp (-\alpha d)]$, which is relevant here since thin films are used [44]. The temporal evolution of the laser pulse intensity $I_{0}$ is described by 


$$
I_{0}(t)=\frac{2 F \sqrt{\ln 2}}{\sqrt{\pi} \tau_{p}} \exp \left[-4 \ln 2\left(t / \tau_{p}\right)^{2}\right]
$$

Here, $F$ and $\tau_{p}$ are the laser fluence and pulse duration, respectively. The system is numerically solved using the following assumptions and boundary conditions. Superheating might occur, resulting in melting time scales of up to $100 \mathrm{ps}$ [46]. Since these time scales are still significantly shorter than the ejection time scale of our LIFT experiments, temperature homogenization is expected to occur prior to ejection. Therefore, superheating is ignored in the temperature model. Heat conduction into the carrier and the air are ignored (i.e., at $z=0$ and $z=d$, we use $\left.C_{e} \frac{\delta T_{e}}{\delta t}=0\right)$. A one-dimensional model is used, because (1) the laser spot size exceeds the film thickness by 2 orders of magnitude and (2) the thermal penetration length in the lateral dimension of the film remains much smaller than the spot size until $\sim 10 \mathrm{~ns}$ after the laser pulse, during which time the material is ejected. The ballistic motion of electrons is ignored, as $\tau_{p}>1 \mathrm{ps}$ [47]. The equations are solved numerically for a time period of $100 \mathrm{ps}$.

\section{RESULTS}

\section{A. Ejection regime classification}

Figure 2 shows the fluence-resolved ejection dynamics for a 200-nm copper film. Figures 2(a)-2(c) show typical ejections in the low-fluence regime $\left(F \leq 600 \mathrm{~mJ} / \mathrm{cm}^{2}\right)$, where the ejection of a cap is observed. For intermediate fluences $\left(600 \mathrm{~mJ} / \mathrm{cm}^{2} \leq F \leq 740 \mathrm{~mJ} / \mathrm{cm}^{2}\right)$, a jetlike feature is formed on the apex of the ejected cap; see Figs. 2(d) and 2 (e). At even higher fluence levels $\left(F \geq 740 \mathrm{~mJ} / \mathrm{cm}^{2}\right)$, ejection of a spray is observed; see Figs. 2(f) and 2(g). These sprays are characterized by a cloud of particles or

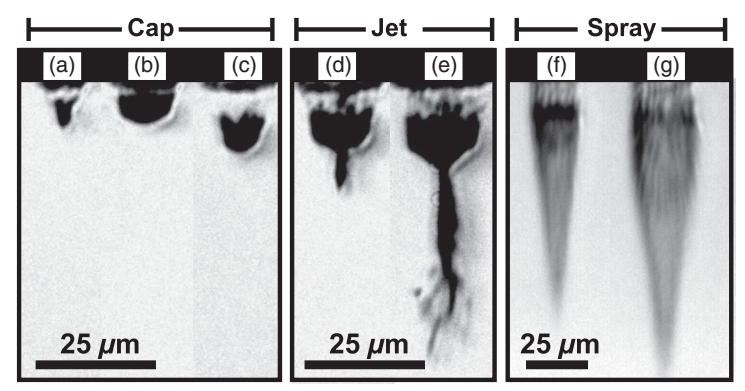

FIG. 2. Ejection of a 200-nm copper film, visualized 125 ns after the incident laser pulse. (a)-(g) correspond to increasing fluence values of $314,392,480,660,864,1060$, and $1576 \mathrm{~mJ} / \mathrm{cm}^{2}$, respectively. The black bar at the top of the image schematically shows the location of the donor layer. (a)-(c) illustrate the cap-ejection regime observed for low fluences; (d),(e) show the formation of a jet from the apex of the cap for intermediate fluences; and (f),(g) show the ejection of a fast copper spray. In (g), some droplets are visible as lines, because their high velocity results in strong motion blur (the tip speed is $700 \mathrm{~m} / \mathrm{s}$ ).

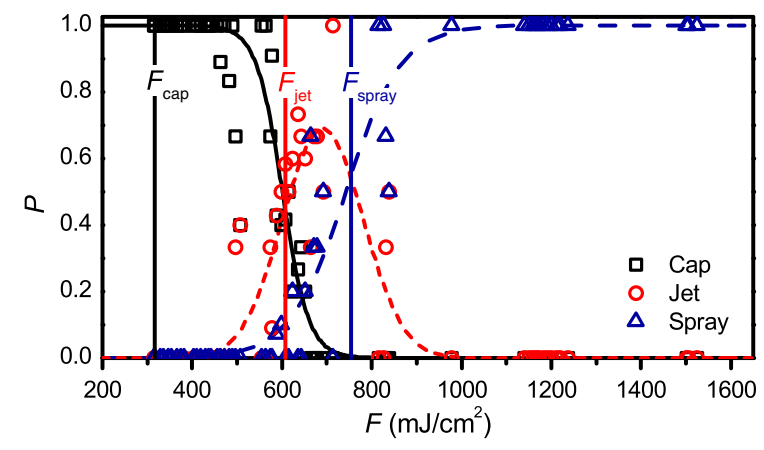

FIG. 3. Probability $P$ of the type of ejections observed (cap, jet, or spray) versus the incident laser fluence.

droplets, instead of the more coherent features observed in the cap-ejection regime. Snapshots of the sometimes spectacular ejection dynamics of each regime are provided in the Supplemental Material for gold [48].

Establishing the threshold fluences between these regimes proved challenging, as experiments with the same input parameter settings sometimes resulted in different regimes (in particular, close to the transition fluences). Therefore, the incidence rate of each regime is binned as a function of fluence. For the 200-nm copper film, 388 ejection events are categorized and binned (using a bin width of $10 \mathrm{~mJ} / \mathrm{cm}^{2}$ ). Figure 3 shows the probability of each ejection regime for each bin. The lower fluence thresholds for the cap, jet, and spray regimes are based on a regime incidence of 50\%, yielding values of 320, 600, and $740 \mathrm{~mJ} / \mathrm{cm}^{2}$, respectively. These thresholds are connected to the film temperature (discussed next), providing evidence for the ejection driving mechanism.

\section{B. Temperature analysis}

The temporal evolution and spatial distribution of the electron and lattice temperatures are computed with the model described in Sec. II. Example results are plotted in Fig. 4, showing the interface lattice temperatures and the electron temperature as a function of time. First, the laser pulse is absorbed by the electron subsystem in the optical absorption depth, which has a thickness of $1 / \alpha \approx 15 \mathrm{~nm} \ll d$, resulting in electron temperatures up to $\sim 10^{4} \mathrm{~K}$ at the carrier-donor interface (red squares in Fig. 4). Subsequently, the lattice is heated by the electrons and reaches a peak temperature of $\sim 2600 \mathrm{~K}$ on the relaxation time of the electron-phonon system $\left(\tau_{\mathrm{ep}} \sim 20 \mathrm{ps}\right)$. A nearly homogeneous temperature is reached after $t \approx 100 \mathrm{ps,} \mathrm{cor-}$ responding to the thermal diffusion time scale $\tau_{\text {th }}=$ $d^{2} /\left(2 \alpha_{D}\right) \approx 180$ ps, with $\alpha_{D}=1.1 \times 10^{-4} \mathrm{~m}^{2} / \mathrm{s}$ the thermal diffusivity.

Figure 5 shows the calculated maximum interface temperatures of the metal film as a function of the laser fluence. The temperature plateaus at $T \approx 1400 \mathrm{~K}$ and $T \approx$ $2800 \mathrm{~K}$ indicate the melting and evaporation phase changes, respectively. Here, increasing the fluence only 


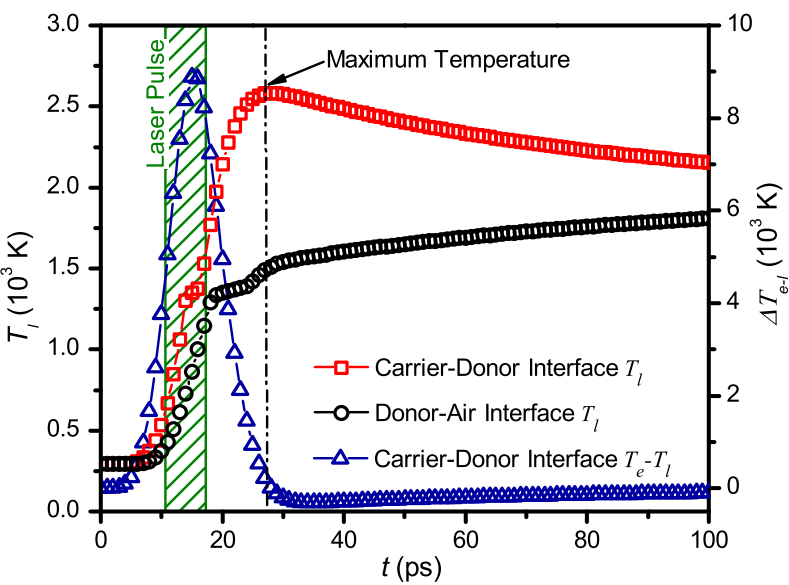

FIG. 4. Calculated lattice temperature at the carrier-donor interface (squares, left axis) and the donor-air interface (circles, left axis) as a function of time (at $t=0$, the simulation starts; the peak pulse energy is reached at $t=2 \tau_{p}=13 \mathrm{ps}$ ), for $F=376 \mathrm{~mJ} / \mathrm{cm}^{2}$. The hashed area indicates the full width half maximum of the laser pulse duration. The electron-phonon temperature difference at the carrier-donor interface (triangles, right axis) illustrates the high electron temperatures reached and the electron cooling on the electron-phonon temperature relaxation time scale ( 20 ps).

results in the phase change of a larger material fraction. The onset of melting (i.e., partial melting of metal close to the carrier-metal interface) is predicted to occur at $F \approx 120 \mathrm{~mJ} / \mathrm{cm}^{2}$, until at a fluence of $F \approx 350 \mathrm{~mJ} / \mathrm{cm}^{2}$, the film is completely melted. The onset of evaporation (i.e., partial evaporation of metal close to the carrier-metal interface) occurs at $F \approx 400 \mathrm{~mJ} / \mathrm{cm}^{2}$. For fluences $\geq 610 \mathrm{~mJ} / \mathrm{cm}^{2}$, the full layer has reached the steady-state vaporization temperature and increasing the fluence only results in a larger vaporized fraction of the film. A further temperature increase is expected only for fluences resulting

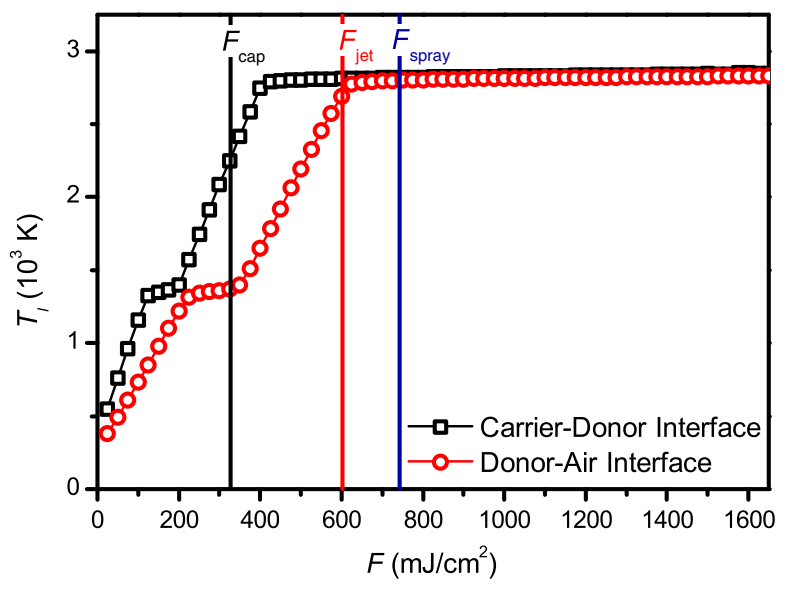

FIG. 5. Computed maximum temperature as a function of laser fluence, at the interfaces of a 200-nm copper film. in complete film vaporization, which are beyond the current parameter space.

The transition fluences, separating the ejection regimes, are replotted in Fig. 5. The ejection threshold for cap ejection $F_{\text {cap }}=320 \mathrm{~mJ} / \mathrm{cm}^{2}$ coincides with the full melting of the donor film. The transition to the jet-ejection regime occurs when the full layer reaches the evaporation temperature (in Sec. IIIC, we argue that the driving mechanisms of the jet and the spray are equal).

This quantitative correspondence between the phase changes and the threshold fluences for cap and jet ejection suggests two different ejection mechanisms, as will be discussed in the next section as follows:

(1) Cap ejection by thermally induced stress relaxation [Sec. III C 1].

(2) No ejection for subthreshold fluences, due to surface tension retracting the cap [Sec. III C 2].

(3) Jet and spray ejection by partial film vaporization [Sec. III C 3].

\section{Ejection mechanisms}

\section{Cap ejection by thermally induced stress relaxation}

As shown in Fig. 5, vaporization is not predicted by our temperature model just above the cap-ejection-fluence threshold. Therefore, thermal compression of the metal film and subsequent release of elastic energy is proposed as the driving mechanism analogous to Refs. [34,38]. The elastic energy is modeled as

$$
E_{E}=\int_{0}^{d} A K u^{2} d z \approx \frac{1}{2} d A K \alpha_{\mathrm{th}}^{2} \Delta T^{2},
$$

using thermal expansion $u=\alpha_{\mathrm{th}} \Delta T$ with $\alpha_{\mathrm{th}}$ the thermal expansion coefficient and $\Delta T$ the lattice temperature increase by the laser pulse, a bulk modulus $K=\frac{E}{3(1-2 \nu)}$ with Young's modulus $E$ and the Poisson ratio $\nu$, and a surface area $A$. The temperature increase is modeled as

$$
\Delta T=\frac{(1-R) F-d H_{m}}{d C_{l}},
$$

with $H_{m}$ the melting enthalpy, $R$ the reflection coefficient, and $C_{l}$ the lattice heat capacity. By equating this elastic energy to the kinetic energy,

$$
E_{\mathrm{kin}}=\frac{1}{2} \rho d A v^{2},
$$

where $\rho$ is the density, the velocity in the elastic regime is derived as

$$
v=\alpha_{\mathrm{th}} C_{0} \Delta T \sqrt{\frac{K}{\rho}},
$$



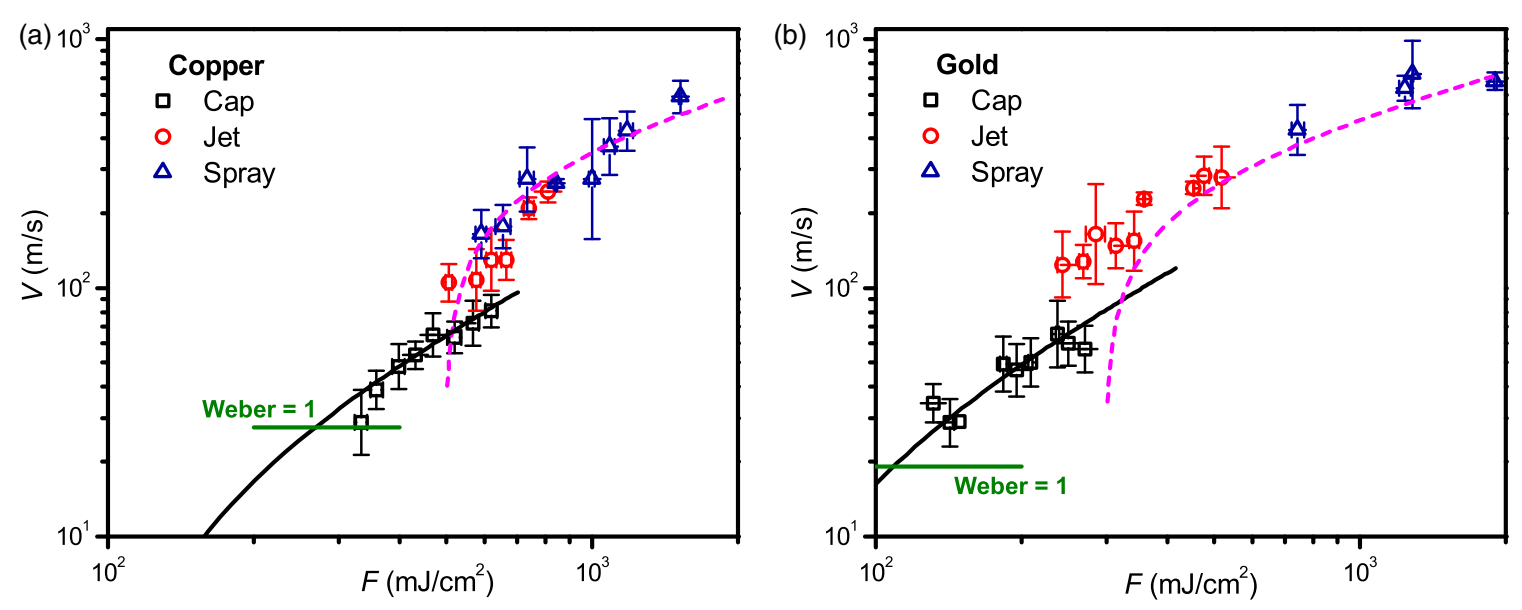

FIG. 6. Ejection velocity as a function of the laser fluence, for a 200-nm copper film (a) and a 160-nm gold film (b). The different markers indicate the ejection regimes analogous to Fig. 2. The green dash-dotted line indicates the predicted minimum velocity; the black solid lines show the modeled cap-ejection velocity [Eq. (8)], and the dashed purple line indicates the spray-and jet-velocity model [Eq. (12)]. The displayed data points represent grouped measurements with error bars indicating the standard deviation (each data point consists of at least 10 individual measurements).

with $C_{0}$ a fitting prefactor. The resulting velocities are shown by the solid lines in Fig. 6(a) for copper (with $C_{0}=0.35$ ) and Fig. 6(b) for gold (with $C_{0}=0.7$ ). Using prefactors of order 1, good agreement between the model and the measurements is obtained.

\section{Threshold ejection fluence governed by the capillary-inertial-energy balance}

Surprisingly, only velocities exceeding $\sim 20 \mathrm{~m} / \mathrm{s}$ are observed even just above the ejection threshold, as shown in Fig. 6. Previous reports have shown that for subejection threshold fluences, the film is accelerated, but retracted by surface tension before it can escape the (liquid) donor layer [22]. This mechanism suggests that ejection only takes place if the kinetic energy of the ejected material exceeds its surface energy. Dividing the kinetic energy $E_{\text {kin }}$ by the surface energy $E_{s}$ provides

$$
\frac{E_{\mathrm{kin}}}{E_{s}} \sim \frac{\rho d v^{2}}{\sigma}=\mathrm{We}
$$

with $v$ the maximum tip velocity, $\sigma$ the surface tension of molten copper, and We the Weber number, i.e., the ratio of inertial energy and surface energy. Using a threshold Weber number $\mathrm{We}=1$, the minimal ejection velocity is readily determined from Eq. (9). As shown in Fig. 6, this velocity reasonably matches our measured minimum velocities.

Using the condition $\mathrm{We}=1$, the minimum cap-ejection fluence is readily determined. Combining Eqs. (6), (8), and (9), and solving for the fluence provides

$$
F_{\text {cap }}=\frac{1}{1-R}\left[\frac{\rho d C_{l}}{\alpha_{\mathrm{th}} C_{0}} \sqrt{\frac{\sigma}{K d}}+d H_{m}\right]
$$

where again for gold $C_{0}=0.7$ and for copper $C_{0}=0.35$ are used. The threshold fluence is plotted as a function of the film thickness in Fig. 7, showing good agreement with the measured data for copper and gold films of various thicknesses. This agreement suggests that the condition $\mathrm{We}=1$ provides a simple and robust criterion for determination of the threshold fluence for LIFT of (locally) liquid-metal films.

\section{Jet and spray ejection by partial film vaporization}

For both jet and spray ejection, vaporization is predicted within the whole film (see Fig. 5). Therefore, vapor-driven ejection is assumed for these regimes [49]. The laser energy heating the vapor is estimated as the initial energy minus

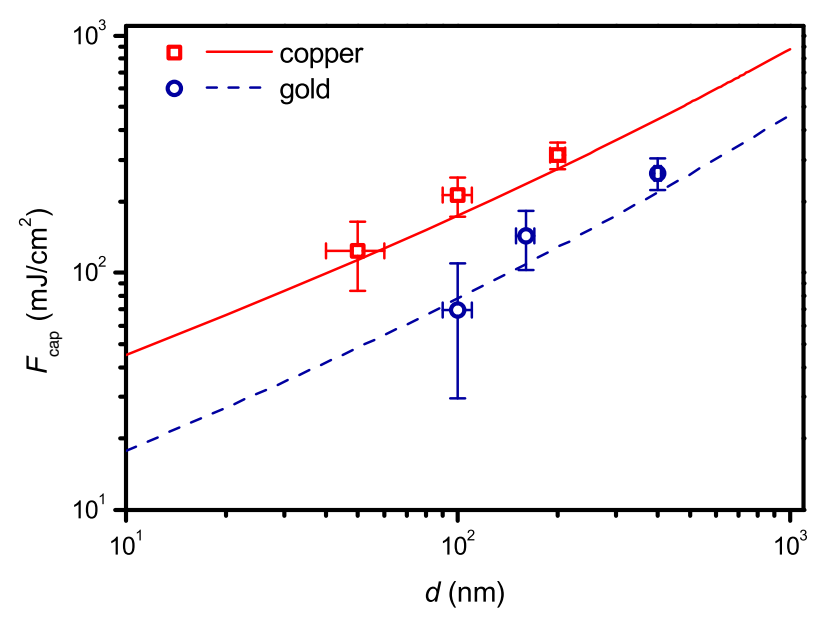

FIG. 7. Threshold ejection fluence $F_{\text {cap }}$ as a function of the film thickness, for copper and gold films. The markers indicate measured values; the lines indicate the model prediction [Eq. (10)]. 
the energy required for melting the film and heating it to the boiling temperature:

$$
E_{\text {vap }}=C_{1} A\left[(1-R) F-d H_{m}-d C_{l}\left(T_{v}-T_{0}\right)\right],
$$

where $T_{0}$ is the initial (room) temperature, $T_{v}$ is the boiling temperature, and $C_{1}$ is a prefactor. Equating the energy contained by the vapor [Eq. (12)] to the kinetic energy [Eq. (7)], the ejection velocity is obtained,

$$
v=\sqrt{\frac{2 C_{1}\left[(1-R) F-d H_{m}-d C_{l}\left(T_{v}-T_{0}\right)\right]}{\rho d} .}
$$

Figure 6 shows the predicted velocities (purple dashed lines) for copper [Fig. 6(a)], using $C_{1}=0.05$ and gold [Fig. 6(b)], using $C_{1}=0.12$. Although prefactors are required for quantitative agreement, the transition from the cap- to the jet-ejection regime (which is hardly influenced by the prefactor) is captured. The nonunity value of $C_{1}$ may be due to a subtlety of the vaporization enthalpy, which partly consists of the energy required for the atom-by-atom escape through the liquid surface, and partly of the work done by the expanding vapor [50]. At room conditions, the work done by the expanding vapor (which we consider as the driving mechanism) is only $8 \%$ of the total vaporization enthalpy for both copper and gold, i.e., of similar order as the prefactors used. Therefore, using only the work done by the expanding vapor (as, for example, provided in Ref. [50]) may allow for a reasonable velocity estimate.

In the spray-ejection regime, the speed of sound in air is generally exceeded by the ejected material, and ejectioninduced shock waves must be present. These shock waves were observed only occasionally and were faint (not shown here). Since the shock waves do not seem to influence the ejection regime or velocity, and detailed visualization requires a different experimental setup, we refer to Refs. [51,52] for a detailed discussion of shock waves.

\section{DISCUSSION}

Liquid-phase cap ejection is a scarcely described ejection regime in LIFT [53], which we therefore concisely discuss. Cap ejection strongly resembles a nanobump torn off around its base [54-56] (a nanobump is a smooth bump in the metal film, which is observed below the ejectionthreshold fluence), which is distinctly different from the commonly reported LIFT ejection by formation and breakup of a liquid filament [17,40]. The dynamics of liquid-phase nanobump formation were recently investigated using molecular dynamics simulations, revealing extreme thinning of the donor film at the base of the bump [38]. Cap ejection was not reported there, since the simulations were limited to short time scales or subejection fluences. However, rupture of this thin part of the film is easily conceivable and would directly correspond to our cap ejections. Because liquid-film rupture is likely to result in the formation of multiple droplets, this mechanism may also explain the generally observed formation of satellite droplets [53]. Despite the multidroplet formation, the capejection regime has a high potential for 3D additive manufacturing, since the ejected cap contracts into a main droplet with a well-defined size and speed.

The cap-ejection threshold fluence is accurately captured by the capillary-inertial-energy balance resulting in Eq. (5). The generality of this energy balance is now assessed using the (rare) literature data on the liquid-film LIFT ejection. These references include velocity data below and above the ejection threshold. First, for numerical work on LIFT of Newtonian liquid films deposited on a dynamic release layer [22], a threshold ejection Weber number $\mathrm{We}_{\text {th }}=1.1$ is obtained in agreement with the $\mathrm{We}=1$ criterion proposed here. In a second case [19], no detachment was observed even for experiments performed at $\mathrm{We} \approx 80$. However, in that case, rheological modifiers were added to the viscous film, potentially delaying film breakup as compared to Newtonian fluids. This difference suggests that $\mathrm{We}=1$ sets the lower bound of the minimum ejection velocity: for $\mathrm{We}<1$, retraction is expected, whereas for We $>1$, ejection occurs only if the film breaks up on a sufficiently short time scale.

Elastic stress release and partial vaporization of the film are well-established driving mechanisms for metal LIFT $[33,34,36-38,44]$. However, the actual driving mechanisms are more complex. For example, numerical simulations revealed that the cap-ejection regime is the result of a heat-induced (but still elastic) pressure wave traveling perpendicular to the film $[36-38,44]$. This wave is reflected at the donor-air surface and induces pull-off of the donor from the carrier [57]. Also, superheating and vapor formation, expansion, and condensation could occur [58]. In this view, it is remarkable that our simple energy balances seem to capture these phenomena, although a prefactor is required for quantitative agreement to the measurements.

Still, even for a single driving mechanism, actual observations may depend on the pulse duration and the film thickness. For example, for nanosecond pulse durations, film deformation and thermal diffusion are significant already during the pulse. Therefore, the energy deposition will be less confined and shock waves within the film could be diminished, resulting in lower ejection velocities [17,59]. For femtosecond pulses, the energy absorption by the electrons is much faster than for our picosecond pulses. However, in both cases, the energy transfer into the lattice is limited by the time scale at which the hot electrons heat the lattice (i.e., the electron-phonon coupling time scale), which usually is on the order of $\sim 10$ ps. Therefore, in this short-pulse regime, lattice heating is hardly affected [60] and our models are expected to be valid. Similarly, the thickness of the film may strongly 
affect the observed ejection regime. In particular, if the optical penetration length is smaller than the film thickness and ejection occurs prior to thermal diffusion over the film thickness, solid-state ejection is observed [25]. In these cases, the ejection threshold depends on the yield strength of the film or its adhesion to the carrier substrate [33,34], and Eq. (5) no longer applies.

\section{CONCLUSIONS}

High-resolution images of donor ejection during picosecond LIFT are presented. Varying the fluence $F$ reveals different ejection regimes, which are illustrated for a $200 \mathrm{~nm}$ copper film. For $320 \mathrm{~mJ} / \mathrm{cm}^{2}<F<600 \mathrm{~mJ} / \mathrm{cm}^{2}$, ejection of a hemispherical piece of the film is observed. This is a scarcely addressed regime that we call cap ejection. For $600 \mathrm{~mJ} / \mathrm{cm}^{2}<F<740 \mathrm{~mJ} / \mathrm{cm}^{2}$, jet ejection occurs, since here a narrow jet leading the cap apex is observed. For $F>740 \mathrm{~mJ} / \mathrm{cm}^{2}$, a cloud of particles is observed, called spray ejection. Using the two-temperature model, these regimes are connected to phase changes within the donor layer. In the cap-ejection regime, the relaxation of elastic stresses within the (melted) donor film is proposed as the driving mechanism. In the jet- and spray-ejection regimes, for which the driving mechanism is similar, the expansion of a vapor bubble drives the ejection. These mechanisms are captured by energy balances which provide velocity predictions. Good agreement with velocity measurements for copper and gold films is obtained, using a materialdependent fitting constant. A minimal ejection velocity of $\approx 20 \mathrm{~m} / \mathrm{s}$ is observed, corresponding to a Weber number $\mathrm{We} \approx 1$. For lower ejection velocities (for which $\mathrm{We}<1$ ), surface tension retracts the liquid film. If the velocity prefactor (which is independent of the film thickness) is known, the $\mathrm{We}=1$ criterion allows the quantitative determination of the ejection threshold fluence. Conversely, if the ejection threshold fluence is known, the velocity can be quantitatively determined.

The current characterization of different LIFT ejection regimes may allow for more controlled deposition of micron-sized pure-metal droplets. In particular, the novel cap-ejection regime may extend the range of achievable droplet sizes and velocities, which we expect to explore in future work.

\section{ACKNOWLEDGMENTS}

The authors thank Andrea Prosperetti, Sander Wildeman, and Roger Jeurissen for fruitful discussions. R.P., G.-W.R., and A.J. H.i.t. V. are grateful to the European Union Seventh Framework Programme for the funding under Grant Agreement No. 260079. C. W. V., C. S., and D. L. acknowledge Fundamenteel Onderzoek der Materie (FOM) and the European Research Council (ERC) for funding.

R. P. and C. W. V. contributed equally to this work.
[1] J. Bohandy, B. F. Kim, and F. J. Adrian, Metal deposition from a supported metal film using an excimer laser, J. Appl. Phys. 60, 1538 (1986).

[2] P. Mogyorósi, T. Szörényi, K. Bali, Zs. Tóth, and I. Hevesi, Pulsed laser ablative deposition of thin metal films, Appl. Surf. Sci. 36, 157 (1989).

[3] Z. Tóth, T. Szörényi, and A. L. Tóth, $\mathrm{Ar}^{+}$laser-induced forward transfer (LIFT): A novel method for micrometersize surface patterning, Appl. Surf. Sci. 69, 317 (1993).

[4] V. Schultze and M. Wagner, Blow-off of aluminum films, Appl. Phys. A 53, 241 (1991).

[5] H. Esrom, J.-Y. Zhang, U. Kogelschatz, and A. J. Pedraza, New approach of a laser-induced forward transfer for deposition of patterned thin metal films, Appl. Surf. Sci. 86, 202 (1995).

[6] D. A. Willis and V. Grosu, Microdroplet deposition by laserinduced forward transfer, Appl. Phys. Lett. 86, 244103 (2005).

[7] I. Zergioti, S. Mailis, N. A. Vainos, P. Papakonstantinou, C. Kalpouzos, C.P. Grigoropoulos, and C. Fotakis, Microdeposition of metal and oxide structures using ultrashort laser pulses, Appl. Phys. A 66, 579 (1998).

[8] D. P. Banks, C. Grivas, J. D. Mills, R. W. Eason, and Ionna Zergioti, Nanodroplets deposited in microarrays by femtosecond Ti:sapphire laser-induced forward transfer, Appl. Phys. Lett. 89, 193107 (2006).

[9] C. Germain, L. Charron, L. Lilge, and Y. Y. Tsui, Electrodes for microfluidic devices produced by laser induced forward transfer, Appl. Surf. Sci. 253, 8328 (2007).

[10] G. Oosterhuis, A. Prenen, and A. J. Huis in 't Veld, Laser induced forward transfer of interconnects for 3D integration, ECS Trans. 41, 81 (2012).

[11] F. Roozeboom, M. Smets, B. Kniknie, M. Hoppenbrouwers, G. Dingemans, W. Keuning, W. M. M. Kessels, R. Pohl, and A. J. Huis in 't Veld, in Proceedings of the 46th IMAPS International Symposium on Microelectronics (International Microelectronics and Packaging Society, Research Triangle Park, NC, 2013).

[12] A. Piqué, D. B. Chrisey, R. C. Y. Auyeung, J. Fitz-Gerald, H. D. Wu, R. A. McGill, S. Lakeou, P. K. Wu, V. Nguyen, and M. Duignan, A novel laser transfer process for direct writing of electronic and sensor materials, Appl. Phys. A 69 , S279 (1999).

[13] B. Hopp, T. Smausz, Zs. Antal, N. Kresz, Zs. Bor, and D. Chrisey, Absorbing film assisted laser induced forward transfer of fungi (trichoderma conidia), Appl. Phys. 96, 3478 (2004).

[14] G. B. Blanchet, Y.-L. Loo, J. A. Rogers, F. Gao, and C. R. Fincher, Large area, high resolution, dry printing of conducting polymers for organic electronics, Appl. Phys. Lett. 82, 463 (2003).

[15] Z. Kántor and T. Szörényi, Dynamics of long-pulse laser transfer of micrometer-sized metal patterns as followed by time-resolved measurements of reflectivity and transmittance, Appl. Phys. 78, 2775 (1995).

[16] H. Fukumura, Y. Kohji, K.-I. Nagasawa, and H. Masuhara, Laser implantation of pyrene molecules into poly(methyl methacrylate) films, J. Am. Chem. Soc. 116, 10304 (1994).

[17] M. P. Giesbers, M. B. Hoppenbrouwers, E. C. P. Smits, and R. Mandamparambil, Process optimization of LIFT through 
visualization, towards high resolution metal circuit printing, Proc. SPIE Int. Soc. Opt. Eng. 9135, 91350Z (2014).

[18] M. Versluis, High-speed imaging in fluids, Exp. Fluids 54, 1458 (2013).

[19] D. Young, R. C. Y. Auyeung, A. Piqué, D. B. Chrisey, and D. D. Dlott, Plume and jetting regimes in a laser based forward transfer process as observed by time-resolved optical microscopy, Appl. Surf. Sci. 197-198, 181 (2002).

[20] M. Duocastella, J. M. Fernández-Pradas, P. Serra, and J. L. Morenza, Jet formation in the laser forward transfer of liquids, Appl. Phys. A 93, 453 (2008).

[21] M. Duocastella, J. M. Fernández-Pradas, J. L. Morenza, and P. Serra, Time-resolved imaging of the laser forward transfer of liquids, J. Appl. Phys. 106, 084907 (2009).

[22] M. S. Brown, C. F. Brasz, Y. Ventikos, and C. B. Arnold, Impulsively actuated jets from thin liquid for high-resolution printing applications, J. Fluid Mech. 709, 341 (2012).

[23] C. Boutopoulos, I. Kalpyris, E. Serpetzoglou, and I. Zergioti, Laser-induced forward transfer of silver nanoparticle ink: Time-resolved imaging of the jetting dynamics and correlation with the printing quality, Microfluid. Nanofluid. 16, 493 (2014).

[24] R. Fardel, M. Nagel, F. Nüesch, T. Lippert, and A. Wokaun, Laser-induced forward transfer of organic LED building blocks studied by time-resolved shadowgraphy, J. Phys. Chem. C 114, 5617 (2010).

[25] M. Domke, S. Rapp, M. Schmidt, and H. P. Huber, Ultrafast movies of thin-film laser ablation, Appl. Phys. A 109, 409 (2012).

[26] D. Bartl, M. Ametowobla, F. Schmid, A. Letsch, M. Hafner, S. Nolte, and A. Tünnermann, Probing timescales during back side ablation of molybdenum thin films with optical and electrical measurement techniques, Opt. Express 21, 16431 (2013).

[27] M. Feinaeugle, A. P. Alloncle, Ph. Delaporte, C. L. Sones, and R. W. Eason, Time-resolved shadowgraph imaging of femtosecond laser-induced forward transfer of solid materials, Appl. Surf. Sci. 258, 8475 (2012).

[28] S. A. Mathews, R. C. Y. Auyeung, H. Kim, N. A. Charipar, and A. Piqué, High-speed video study of laser-induced forward transfer of silver nano-suspensions, J. Appl. Phys. 114, 064910 (2013).

[29] Y. Nakata and T. Okada, Time-resolved microscopic imaging of the laser-induced forward transfer process, Appl. Phys. A 69, S275 (1999).

[30] T. Sano, H. Yamada, T. Nakayama, and I. Miyamoto, Experimental investigation of laser induced forward transfer process of metal thin films, Appl. Surf. Sci. 186, 221 (2002).

[31] A. B. Bullock and P. R. Bolton, Laser-induced back ablation of aluminum thin films using picosecond laser pulses, J. Appl. Phys. 85, 460 (1999).

[32] I. Zergioti, D. G. Papazoglou, A. Karaiskou, C. Fotakis, E. Gamaly, and A. Rode, A comparative Schlieren imaging study between ns and sub-ps laser forward transfer of $\mathrm{Cr}$, Appl. Surf. Sci. 208-209, 177 (2003).

[33] B. Tóth, Z. Hopp, T. Szörényi, Z. Bor, E. A. Shakhno, and V. P. Veiko, in Proceedings of SPIE, edited by V. P. Veiko and T. Szoerenyi, Vol. 3822 (SPIE, Bellingham, WA, 1999), p. 18-26.
[34] V. P. Veiko, E. A. Shakhno, V. N. Smirnov, A. M. Miaskovski, and G. D. Nikishin, Laser-induced film deposition by lift physical mechanisms and applications, Laser Part. Beams 24, 203 (2006).

[35] C. Unger, M. Grüne, L. Koch, J. Koch, and B. N. Chichkov, Time-resolved imaging of hydrogel printing via laser-induced forward transfer, Appl. Phys. A 103, 271 (2011).

[36] D. S. Ivanov and L. V. Zhigilei, Combined atomisticcontinuum modeling of short-pulse laser melting and disintegration of metal films, Phys. Rev. B 68, 064114 (2003).

[37] C. Mézel, L. Hallo, A. Souquet, J. Breil, D. Hébert, and F. Guillemot, Self-consistent modeling of jet formation process in the nanosecond laser pulse regime, Phys. Plasmas 16, 123112 (2009).

[38] D. S. Ivanov, A. I. Kuznetsov, V. P. Lipp, B. Rethfeld, B. N. Chichkov, M. E. Garcia, and W. Schulz, Short laser pulse nanostructuring of metals: Direct comparison of molecular dynamics modeling and experiment, Appl. Phys. A 111, 675 (2013).

[39] T. C. Röder and J. R. Köhler, Physical model for the laser induced forward transfer process, Appl. Phys. Lett. 100, 071603 (2012).

[40] A. I. Kuznetsov, C. Unger, J. Koch, and B. N. Chichkov, Laser-induced jet formation and droplet ejection from thin metal films, Appl. Phys. A 106, 479 (2012).

[41] J. M. Liu, Simple technique for measurements of pulsed Gaussian-beam spot sizes, Opt. Lett. 7, 196 (1982).

[42] C. W. Visser, P. E. Frommhold, S. Wildeman, R. Mettin, C. Sun, and D. Lohse, Dynamics of high-speed micro-drop impact, numerical simulations and experiments at frame-toframe times below $100 \mathrm{~ns}$, Soft Matter (in press).

[43] S.-S.Wellershoff, J. Hohlfeld, J. Güdde, and E. Matthias, The role of electron-phonon coupling in femtosecond laser damage of metals, Appl. Phys. A 69, S99 (1999).

[44] M. V. Shugaev and N. M. Bulgakova, Thermodynamic and stress analysis of laser-induced forward transfer of metals, Appl. Phys. A 101, 103 (2010).

[45] See the Supplemental Material at http://link.aps.org/ supplemental/10.1103/PhysRevApplied.3.024001, Table S1, for the material properties used.

[46] B. Rethfeld, K. Sokolowski-Tinten, D. von der Linde, and S. I. Anisimov, Ultrafast thermal melting of laser-excited solids by homogeneous nucleation, Phys. Rev. B 65 , 092103 (2002).

[47] J. Hohlfeld, S.-S. Wellershoff, J. Güdde, U. Conrad, V. Jähnke, and E. Matthias, Electron and lattice dynamics following optical excitation of metals, Chem. Phys. 251, 237 (2000).

[48] See the Supplemental Material at http://link.aps.org/ supplemental/10.1103/PhysRevApplied.3.024001, Fig. S1, for snapshots of gold film ejection.

[49] The different footprints of these regimes (observed from Fig. 2) are likely due to the Gaussian spatial distribution of the laser pulse. In the jetting regime, vaporization only occurs in a small spot in the center of the melt area, whereas in the spray-ejection regime, vaporization occurs in almost the full melt area. The similarity of the jet and spray regimes is confirmed by the partial overlap of the jet- and sprayejection velocities (Fig. 6), and therefore no distinction is made regarding the driving mechanism. 
[50] J. Garai, Physical model for vaporization, Fluid Phase Equilib. 283, 89 (2009).

[51] X. Zeng, X. L. Mao, R. Greif, and R. E. Russo, Experimental investigation of ablation efficiency and plasma expansion during femtosecond and nanosecond laser ablation of silicon, Appl. Phys. A 80, 237 (2005).

[52] R. Fardel, M. Nagel, F. Nüesch, T. Lippert, and A. Wokaun, Laser-induced forward transfer of organic LED building blocks studied by time-resolved shadowgraphy, J. Phys. Chem. C 114, 5617 (2010).

[53] R. Pohl, C. W. Visser, G. Römer, C. Sun, and D. Lohse (to be published).

[54] F. Korte, J. Koch, and B. N. Chichkov, Formation of microbumps and nanojets on gold targets by femtosecond laser pulses, Appl. Phys. A 79, 879 (2004).

[55] A. I. Kuznetsov, J. Koch, and B. N. Chichkov, Nanostructuring of thin gold films by femtosecond lasers, Appl. Phys. A 94, 221 (2009).

[56] D. A. Willis and V. Grosu, The effect of melting-induced volumetric expansion on initiation of laser-induced forward transfer, Appl. Surf. Sci. 253, 4759 (2007).

[57] Note that stress release towards the nonheated region of the film is limited by the speed of sound. During the laser pulse duration, the radius of the heated area $(10 \mu \mathrm{m})$ significantly exceeds the distance the compression wave travels through the film $(<1 \mu \mathrm{m})$. Therefore, film buckling is not due to lateral compression, but due to (still-elastic [36]) pressurewave reflection. These reflections have been incorporated in more comprehensive semianalytical models; see, for example, Ref. [61].

[58] F. J. Adrian, J Bohandy, B. F. Kim, and A. N. Nette, A study of the mechanism of metal deposition by the laser-induced forward transfer process, J. Vac. Sci. Technol. B 5, 1490 (1987).

[59] R. Pohl, C. W. Visser, G. R. B. E. Römer, C. Sun, A. J. Huis in 't veld, and D. Lohse, in Proceedings of LPM, Vilnius, Lithuania, 2014 [Japan Laser Processing Society (JLPS), Osaka, Japan, 2014], pp. 1-5.

[60] B. N. Chichkov, C. Momma, S. Nolte, F. Von Alvensleben, and A. Tünnermann, Femtosecond, picosecond and nanosecond laser ablation of solids, Appl. Phys. A 63, 109 (1996).

[61] R. Fardel, M. Nagel, F. Nüesch, T. Lippert, and A. Wokaun, Energy balance in a laser-induced forward transfer process studied by shadowgraphy, J. Phys. Chem. C 113, 11628 (2009). 